\title{
Low-Order Nonconforming Mixed Finite Element Methods for Stationary Incompressible Magnetohydrodynamics Equations
}

\author{
Dongyang Shi ${ }^{1}$ and Zhiyun $\mathbf{Y u}^{1,2}$ \\ ${ }^{1}$ Department of Mathematics, Zhengzhou University, Zhengzhou 450052, China \\ ${ }^{2}$ College of Science, Zhongyuan University of Technology, Zhengzhou 450007, China \\ Correspondence should be addressed to Dongyang Shi, shi_dy@zzu.edu.cn
}

Received 14 February 2012; Accepted 2 April 2012

Academic Editor: Livija Cveticanin

Copyright (C) 2012 D. Shi and Z. Yu. This is an open access article distributed under the Creative Commons Attribution License, which permits unrestricted use, distribution, and reproduction in any medium, provided the original work is properly cited.

\begin{abstract}
The nonconforming mixed finite element methods (NMFEMs) are introduced and analyzed for the numerical discretization of a nonlinear, fully coupled stationary incompressible magnetohydrodynamics (MHD) problem in 3D. A family of the low-order elements on tetrahedra or hexahedra are chosen to approximate the pressure, the velocity field, and the magnetic field. The existence and uniqueness of the approximate solutions are shown, and the optimal error estimates for the corresponding unknown variables in $L^{2}$-norm are established, as well as those in a broken $H^{1}$ norm for the velocity and the magnetic fields. Furthermore, a new approach is adopted to prove the discrete Poincaré-Friedrichs inequality, which is easier than that of the previous literature.
\end{abstract}

\section{Introduction}

This work deals with the numerical discretization of a nonlinear, fully coupled stationary incompressible MHD problem by a family of the low-order NMFEMs. This requires discretizing a system of partial differential equations that couples the incompressible NavierStokes equations with Maxwell's equations.

The MHD problem has a number of applications such as liquid-metal cooling of nuclear reactors, electromagnetic casting of metals, MHD power generation, and MHD ion propulsion (cf. $[1,2])$. Thus, many studies have already been devoted to the MHD problem. For theoretical results, let us just mention those by [3-5]. It is important to employ effective numerical methods to approximate the exact solutions of the MHD problem because the exact solutions can be obtained only for some special cases [2]. Compared with the finite difference methods [6-8], most studies are performed by the finite element methods (FEMs) [9-24]. 
Precisely speaking, the work started with [9], where inf-sup stable mixed elements were used to discretize the velocity field and the pressure, and $H^{1}$-conforming elements for the magnetic field, and the existence and uniqueness of the discrete solutions with inhomogeneous boundary condition satisfying certain assumptions were proved and the convergence analysis was presented. In contrast to the results of [9], [10] derived the same results without any restrictions on the boundary data of the velocity field. Reference [11] examined the long-term dissipativity and unconditional nonlinear stability of time integration algorithms for an incompressible MHD problem. Reference [12] dealt with a decoupled linear MHD problem involving electrically conducting and insulating regions by Lagrange finite elements and gave error estimates for a fully discrete scheme. For convex polyhedral domains, or domains with a boundary $C^{1,1}$, the convergence analysis of a stabilized FEM, the optimal control method, and two-level FEMs were investigated in [1315], [16], and [17], respectively.

On the other hand, some different approaches to achieve convergence results in general Lipschitz polyhedral domains were realized. For example, a mixed discrete formulation about the problem based on $H$ (curl)-conforming (edge) elements to approximate the magnetic field was proposed in $[18,19]$. This observation motivated the works such as the least-squares mixed FEM used in [20], the mixed discontinuous Galerkin method employed in $[21,22]$, and the splitting method presented in [23, 24]. However, all the analyses in [9-24] are about the conforming FEMs except [22].

As we know, nonconforming FEMs have certain advantages over conforming FEMs in some aspects. Firstly, the nonconforming elements are much easier to be constructed to satisfy the discrete inf-sup condition. Secondly, nonconforming elements have been used effectively especially in fluid and solid mechanics due to their stability. We refer to [25-34] for more details on the properties of nonconforming elements applied to incompressible flow problems.

For the Stokes equations, $[25,26]$ considered the approximations of nonconforming $P_{1} / P_{0}$ element and the rotated $Q_{1} / Q_{0}$ element and got first-order accuracy, respectively. Reference [27] modified the rotated $Q_{1} / Q_{0}$ element used in [26] and derived the same convergence order as [26]. For the Navier-Stokes equations, [28-30] obtained maximum norm estimates of $P_{1} / P_{0}$ element and the optimal error estimates of $E Q_{1}^{\text {rot }} / Q_{0}$ element both in broken $H^{1}$-norm for the velocity field and in $L^{2}$-norm for the pressure with moving grids and anisotropic meshes. Furthermore, NMFEMs also have been applied to other problems such as the Darcy-Stokes equations [31], the conduction-convection problem [32, 33], and the diffusion-convection-reaction equation [34].

Especially, [22] firstly presented a NMFEM with exactly divergence-free velocities for a incompressible MHD problem where the velocity and the magnetic fields were approximated by divergence-conforming elements and curl-conforming Nédélec elements, respectively, and derived nearly optimal error estimates. Motivated by the ideas of $[22,32,34-36]$, in this paper, we are interested in discretizations for the MHD problem that are based on NMFEMs; a family of the low order elements will be adopted as approximation spaces for the velocity field, the piecewise constant element for the pressure, and the lowest order $H^{1}$-conforming element for the magnetic field on hexahedra or tetrahedra. The existence and uniqueness of the approximate solutions are shown, and the optimal error estimates for the corresponding unknown variables in $L^{2}$-norm are established, as well as those in a broken $H^{1}$-norm for the velocity, and the magnetic fields. Furthermore, a new approach is adopted to prove the discrete Poincaré-Friedrichs inequality, which is easier than that of the previous literature $[37,38]$. 
The organization of this paper is as follows. In Section 2, we introduce the mixed variational formulation for the MHD problem. Section 3 will give the nonconforming mixed finite element schemes. In Section 4, we state some important lemmas and prove the existence and uniqueness of the approximate solutions. In Section 5, the optimal error estimates for the pressure, the velocity and the magnetic fields in $L^{2}$-norm are established, as well as ones in broken $H^{1}$-norm for the velocity and the magnetic fields.

Throughout the paper, $C$ indicates a positive constant, possibly differs at different occurrences, which is independent of the mesh parameter $h$, but may depend on $\Omega$ and other parameters that appeared in this paper. Notations that are not especially explained are used with their usual meanings.

\section{Equations and the Mixed Variational Formulation}

In this section, we will consider a nonlinear, fully coupled stationary incompressible MHD problem in $3 \mathrm{D}$ as follows (see $[9,15])$.

Problem (I). Find the velocity field $u=\left(u_{1}, u_{2}, u_{3}\right)$, the pressure $p$, the magnetic field $B=$ $\left(B_{1}, B_{2}, B_{3}\right)$ such that

$$
\begin{gathered}
-M^{-2} \Delta u+N^{-1} u \cdot \nabla u+\nabla p-R_{m}^{-1}(\nabla \times B) \times B=f \quad \text { in } \Omega, \\
R_{m}^{-1} \nabla \times(\nabla \times B)-\nabla \times(u \times B)=0 \quad \text { in } \Omega, \\
\nabla \cdot u=0 \quad \text { in } \Omega, \\
\nabla \cdot B=0 \quad \text { in } \Omega, \\
u=0 \quad \text { on } \partial \Omega, \\
B \cdot n=0 \quad \text { on } \partial \Omega, \\
(\nabla \times B) \times n=0 \quad \text { on } \partial \Omega,
\end{gathered}
$$

where $\Omega$ is a simply connected, bounded domain with unit outward normal $n=\left(n_{1}, n_{2}, n_{3}\right)$ on $\partial \Omega$. $M, N$, and $R_{m}$ are the Hartman number, interaction parameter, and magnetic Reynolds number, respectively. The symbols $\Delta, \nabla$, and $\nabla \cdot$ denote the Laplace, gradient, and divergence operators, respectively. $\nabla \times(\nabla \times B)=\nabla(\nabla \cdot B)-\Delta B . f \in H^{-1}(\Omega)^{3}$ is the body force.

Set

$$
\begin{gathered}
H_{0}^{1}(\Omega)^{3}=\left\{v \in H^{1}(\Omega)^{3} ;\left.v\right|_{\partial \Omega}=0\right\}, \\
L_{0}^{2}(\Omega)=\left\{q \in L^{2}(\Omega) ; \int_{\Omega} q d \mathbf{x}=0\right\}, \\
H_{n}^{1}(\Omega)^{3}=\left\{v \in H^{1}(\Omega)^{3} ;\left.(v \cdot n)\right|_{\partial \Omega}=0\right\},
\end{gathered}
$$

here and later, $\mathbf{x}=(x, y, z)$. 
The mixed variational formulation for Problem (I) is written as follows.

Problem $\left(\mathbf{I}_{1}\right)$. Find $(u, B) \in W(\Omega), p \in L_{0}^{2}(\Omega)$ such that

$$
\begin{gathered}
a((u, B),(u, B),(v, \Psi))+b((v, \Psi), p)=F((v, \Psi)), \quad \forall(v, \Psi) \in W(\Omega), \\
b((u, B), \chi)=0, \quad \forall \chi \in L_{0}^{2}(\Omega),
\end{gathered}
$$

where

$$
\begin{gathered}
W(\Omega)=H_{0}^{1}(\Omega)^{3} \times H_{n}^{1}(\Omega)^{3}, \\
a((u, B),(v, \Psi),(w, \Phi)):=a_{0}((v, \Psi),(w, \Phi))+a_{1}((u, B),(v, \Psi),(w, \Phi)) \\
a_{0}((v, \Psi),(w, \Phi)):=M^{-2} \int_{\Omega} \nabla v: \nabla w d \mathbf{x}+R_{m}^{-2} \int_{\Omega}[(\nabla \times \Psi) \cdot(\nabla \times \Phi)+(\nabla \cdot \Psi)(\nabla \cdot \Phi)] d \mathbf{x}, \\
a_{1}((u, B),(v, \Psi),(w, \Phi)):=c_{0}(u ; v, w)-c_{1}(B ; w, \Psi)+c_{2}(B ; v, \Phi), \\
c_{0}(u ; v, w):=\int_{\Omega}(2 N)^{-1}(u \cdot \nabla v \cdot w-u \cdot \nabla w \cdot v) d \mathbf{x} \\
c_{1}(B ; w, \Psi):=\int_{\Omega} R_{m}^{-1}(\nabla \times \Psi) \times B \cdot w d \mathbf{x} \\
c_{2}(B ; v, \Phi):=\int_{\Omega} R_{m}^{-1}(\nabla \times \Phi) \times B \cdot v d \mathbf{x} \\
b((v, \Psi), x):=-\int_{\Omega} x \nabla \cdot v d \mathbf{x}, \quad F((v, \Psi)):=\int_{\Omega} f v d \mathbf{x} .
\end{gathered}
$$

It has been shown in $[9,37,38]$ that for $u, v, w \in H_{0}^{1}(\Omega)^{3}, B, \Psi, \Phi \in H_{n}^{1}(\Omega)^{3}$, there hold

$$
\begin{gathered}
c_{0}(u ; v, w)=-c_{0}(u ; w, v), \quad c_{0}(w ; v, v)=0, \\
a_{1}((u, B),(v, \Psi),(w, \Phi))=-a_{1}((u, B),(w, \Phi),(v, \Psi)), \\
a_{1}((u, B),(v, \Psi),(v, \Psi))=0 .
\end{gathered}
$$

Let $Z(\Omega)=\left\{v \in H_{0}^{1}(\Omega)^{3}, \nabla \cdot v=0\right\}$. For $v \in H_{0}^{1}(\Omega)^{3}$ and $\Psi \in H_{n}^{1}(\Omega)^{3}$, we will equip $W(\Omega)$ with the norm

$$
\begin{gathered}
\|(v, \Psi)\|_{W}:=\left(\|v\|_{1}^{2}+\|\Psi\|_{1}^{2}\right)^{1 / 2}, \\
\|f\|_{-1}:=\sup _{(0,0) \neq(v, \Psi) \in W(\Omega)} \frac{f((v, \Psi))}{\|(v, \Psi)\|_{W}}
\end{gathered}
$$

respectively, where $\|\cdot\|_{1}$ is the $H^{1}$-norm. 
The following result can be found in [9].

Theorem 2.1. If $f \in H^{-1}(\Omega)^{3}$, then Problem $\left(\mathbf{I}_{1}\right)$ has at least a solution, in addition, that is unique provided that

$$
C_{2} \gamma_{3}\left(C_{1} \gamma_{1}\right)^{-2}\|f\|_{-1}<1
$$

and satisfying the stability bound

$$
\|(u, B)\|_{W} \leq\left(C_{1} \gamma_{1}\right)^{-1}\|f\|_{-1},
$$

where $\gamma_{1}=\min \left\{M^{-2}, R_{m}^{-2}\right\}, \gamma_{2}=\max \left\{M^{-2}, R_{m}^{-2}\right\}, \gamma_{3}=\max \left\{N^{-1}, R_{m}^{-1}\right\}$ and $C_{1}, C_{2}$ are positive constants only depending on the domain $\Omega$.

\section{Nonconforming Mixed Finite Element Schemes}

Let $\Gamma^{h}=\{K\}$ be regular and quasi-uniform tetrahedra or hexahedra partition of $\Omega$ with mesh size $h$. We use the finite element spaces $X_{1 h} \nsubseteq H_{0}^{1}(\Omega)^{3}, M_{h} \subset L_{0}^{2}(\Omega)$ and $X_{2 h} \subset H_{n}^{1}(\Omega)^{3}$ to approximate the unknown variables $u, p$, and $B$. The following assumptions about the space pair $\left(X_{1 h}, M_{h}\right)$ are provided:

(A) for all $K \in \Gamma^{h}, P_{1}(K)^{3} \subset X_{1 h}$;

(B) $M_{h}=\left\{x^{h} \in L_{0}^{2}(\Omega) ;\left.x^{h}\right|_{K}\right.$ a constant, $\left.\forall K \in \Gamma^{h}\right\}$;

(C) $\|\cdot\|_{1 h}=\left(\sum_{K \in \Gamma^{h}}|\cdot|_{1, K}^{2}\right)^{1 / 2}$ is a norm $X_{1 h}$;

(D) for all $v^{h} \in X_{1 h}, \int_{F}\left[v^{h}\right] d s=0, F \subset \partial K$;

(E) for all $v \in H_{0}^{1}(\Omega)^{3}, q^{h} \in M_{h}, b_{1 h}\left(v-\Pi^{1} v, q^{h}\right)=0,\left\|\Pi^{1} v\right\|_{1 h} \leq C|v|_{1}$, where $\left[v^{h}\right]$ stands for the jump of $v^{h}$ across the face $F$ if $F$ is an internal face, and it is equal to $v^{h}$ itself if $F \subset \partial \Omega, \Pi^{1}$ is the interpolation operator associated with $X_{1 h}$ satisfying $\Pi_{K}=\left.\Pi^{1}\right|_{K}$ for $K \in \Gamma^{h}$, and $P_{1}(K)$ is the polynomial space of degree less than or equal to one on $K$.

Introduce the finite element space

$$
R_{1}(K)= \begin{cases}P_{1}(K) & \text { if } K \text { is tetrahedra } \\ Q_{1}(K) & \text { if } K \text { is hexahedra. }\end{cases}
$$

The finite element space $X_{2 h}$ is defined by

$$
X_{2 h}=\left\{\Psi^{h} \in H_{n}^{1}(\Omega)^{3} ;\left.q^{h}\right|_{K} \in\left(R_{1}(K)\right)^{3},\left.\left(\Psi^{h} \cdot n\right)\right|_{\partial \Omega}=0, \forall K \in \Gamma^{h}\right\},
$$

where $Q_{1}(K)$ is a space of polynomials whose degrees for $x, y, z$ are equal to one. So these are the nonconforming mixed finite element schemes.

Remark 3.1. It can be checked that the nonconforming finite elements studied in [25-33, 3945] satisfy the above assumptions (A)-(E). 


\section{The Existence and Uniqueness of the Approximate Solutions and Some Lemmas}

In this section, we will prove some lemmas and the existence and uniqueness of the discrete solutions of nonconforming mixed finite element approximations for MHD equations.

Let $W_{h}=X_{1 h} \times X_{2 h}$ and the trilinear forms $a_{h}, a_{1 h}, c_{i h}(i=0,1,2)$ and the bilinear forms $a_{0 h}$ and $b_{h}$ be defined as follows:

for $\left(u^{h}, B^{h}\right),\left(v^{h}, \Psi^{h}\right),\left(w^{h}, \Phi^{h}\right) \in W_{h}$ and $x^{h} \in M_{h}$,

$$
\begin{aligned}
& a_{h}\left(\left(u^{h}, B^{h}\right),\left(v^{h}, \Psi^{h}\right),\left(w^{h}, \Phi^{h}\right)\right) \\
& \quad:=a_{0 h}\left(\left(v^{h}, \Psi^{h}\right),\left(w^{h}, \Phi^{h}\right)\right)+a_{1 h}\left(\left(u^{h}, B^{h}\right),\left(v^{h}, \Psi^{h}\right),\left(w^{h}, \Phi^{h}\right)\right), \\
& a_{0 h}\left(\left(v^{h}, \Psi^{h}\right),\left(w^{h}, \Phi^{h}\right)\right) \\
& \quad=\sum_{K \in \Gamma^{h}}\left\{M^{-2} \int_{K} \nabla v^{h} \cdot \nabla w^{h}+R_{m}^{-2} \int_{K}\left[\left(\nabla \times \Psi^{h}\right) \cdot\left(\nabla \times \Phi^{h}\right)+\left(\nabla \cdot \Psi^{h}\right)\left(\nabla \cdot \Phi^{h}\right)\right]\right\} d \mathbf{x},
\end{aligned}
$$

$$
a_{1 h}\left(\left(u^{h}, B^{h}\right),\left(v^{h}, \Psi^{h}\right),\left(w^{h}, \Phi^{h}\right)\right):=c_{0 h}\left(u^{h} ; v^{h}, w^{h}\right)-c_{1 h}\left(B^{h} ; w^{h}, \Psi^{h}\right)+c_{2 h}\left(B^{h} ; v^{h}, \Phi^{h}\right)
$$

$$
\begin{gathered}
c_{0 h}\left(u^{h} ; v^{h}, w^{h}\right):=\sum_{K \in \Gamma^{h}} \int_{K}(2 N)^{-1}\left(u^{h} \cdot \nabla v^{h} \cdot w^{h}-u^{h} \cdot \nabla w^{h} \cdot v^{h}\right) d \mathbf{x}, \\
c_{1 h}\left(B^{h} ; w^{h}, \Psi^{h}\right)=: \sum_{K \in \Gamma^{h}} \int_{K} R_{m}^{-1}\left(\nabla \times \Psi^{h}\right) \times B^{h} \cdot w^{h} d \mathbf{x}, \\
c_{2 h}\left(B^{h} ; v^{h}, \Phi^{h}\right):=\sum_{K \in \Gamma^{h}} \int_{K} R_{m}^{-1}\left(\nabla \times \Phi^{h}\right) \times B^{h} \cdot v^{h} d \mathbf{x} \\
b_{h}\left(\left(v^{h}, \Psi^{h}\right), x^{h}\right):=-\sum_{K \in \Gamma^{h}} \int_{K} x^{h} \nabla \cdot v^{h} d \mathbf{x},
\end{gathered}
$$

respectively.

Then the approximate formulation of Problem $\left(\mathbf{I}_{1}\right)$ reads as follows.

Problem $\left(\mathbf{I}_{2}\right)$. Find $\left(u^{h}, B^{h}\right) \in W_{h}, p^{h} \in M_{h}$ such that for all $\left(v^{h}, \Psi^{h}\right) \in W_{h}, x^{h} \in M_{h}$,

$$
\begin{gathered}
a_{h}\left(\left(u^{h}, B^{h}\right),\left(u^{h}, B^{h}\right),\left(v^{h}, \Psi^{h}\right)\right)+b_{h}\left(\left(v^{h}, \Psi^{h}\right), p^{h}\right)=F\left(\left(v^{h}, \Psi^{h}\right)\right), \\
b_{h}\left(\left(u^{h}, B^{h}\right), x^{h}\right)=0 .
\end{gathered}
$$

From the definition of (4.3), $a_{1 h}$ satisfies the following antisymmetric properties [9]:

$$
\begin{gathered}
a_{1 h}\left(\left(u^{h}, B^{h}\right),\left(v^{h}, \Psi^{h}\right),\left(v^{h}, \Psi^{h}\right)\right)=0, \\
a_{1 h}\left(\left(u^{h}, B^{h}\right),\left(v^{h}, \Psi^{h}\right),\left(w^{h}, \Phi^{h}\right)\right)=-a_{1 h}\left(\left(u^{h}, B^{h}\right),\left(w^{h}, \Phi^{h}\right),\left(v^{h}, \Psi^{h}\right)\right) .
\end{gathered}
$$


Journal of Applied Mathematics

Let $Z_{h}=\left\{v^{h} \in X_{1 h}, b\left(\left(v^{h}, \Psi^{h}\right), X^{h}\right)=0\right\}$. For all $v^{h}=\left(v_{1}^{h}, v_{2}^{h}, v_{3}^{h}\right) \in X_{1 h}, \Psi^{h}=$ $\left(\Psi_{1}^{h}, \Psi_{2}^{h}, \Psi_{3}^{h}\right) \in X_{2 h}$, we define

$$
\begin{gathered}
\left\|v^{h}\right\|_{0 h}=\left(\sum_{K \in \Gamma^{h}}\left\|v^{h}\right\|_{0, K}^{2}\right)^{1 / 2},\left\|v^{h}\right\|_{1 h}=\left(\sum_{K \in \Gamma^{h}}\left|v^{h}\right|_{1, K}^{2}\right)^{1 / 2}, \\
\left\|\left(v^{h}, \Psi^{h}\right)\right\|_{h}=\left(\sum_{K \in \Gamma^{h}}\left\|v^{h}\right\|_{1 h}^{2}+\left\|\Psi^{h}\right\|_{1}^{2}\right)^{1 / 2}, \\
\|f\|_{h}:=\sup _{(0,0) \neq\left(v^{h}, \Psi^{h}\right) \in X_{1 h} \times X_{2 h}} \frac{F\left(\left(v^{h}, \Psi^{h}\right)\right)}{\left\|\left(v^{h}, \Psi^{h}\right)\right\|_{h}}
\end{gathered}
$$

respectively. Then it is easy to see that $\|\cdot\|_{0 h}$ and $\|\cdot\|_{1 h}$ are the norms over $X_{1 h}$ and $\|(\cdot, \cdot)\|_{h}$ is the norm over $W_{h}$.

Lemma 4.1. The following discrete Poincaré-Friedrichs inequality holds:

$$
\left\|\Psi^{h}\right\|_{0} \leq C\left\|\nabla \times \Psi^{h}\right\|_{0^{\prime}} \quad \forall \Psi^{h} \in X_{2 h}
$$

Proof. We consider the following problem:

$$
\begin{gathered}
\nabla \times(\nabla \times \tilde{B})=f \quad \text { in } \Omega, \\
\nabla \cdot \widetilde{B}=0 \quad \text { in } \Omega, \\
\widetilde{B} \cdot n=0 \quad \text { on } \partial \Omega, \\
(\nabla \times \tilde{B}) \times n=0 \quad \text { on } \partial \Omega .
\end{gathered}
$$

Then by [3], the solution $\widetilde{B}$ of (4.12) satisfies

$$
\|\nabla \times \widetilde{B}\|_{0} \leq C\|f\|_{0}
$$

On the one hand, by Green's formula and Hölder's inequality, we deduce that

$$
\begin{aligned}
\left|\int_{\Omega} f \Psi^{h} d \mathbf{x}\right| & =\left|\sum_{K \in \Gamma^{h}} \int_{K}(\nabla \times \widetilde{B})\left(\nabla \times \Psi^{h}\right) d \mathbf{x}\right| \\
& \leq\|\nabla \times \widetilde{B}\|_{0}\left\|\nabla \times \Psi^{h}\right\|_{0} .
\end{aligned}
$$

Using (4.13)-(4.14) and choosing $f=\Psi^{h}$, we may get the desired result.

Remark 4.2. The method used in this lemma is different from and easier than that of $[37,38]$. 
Lemma 4.3. For $\left(u^{h}, B^{h}\right),\left(v^{h}, \Psi^{h}\right)$, and $\left(w^{h}, \Phi^{h}\right) \in W_{h}$, we have
(1) $\left|\mathcal{C}_{0 h}\left(u^{h} ; v^{h}, w^{h}\right)\right| \leq C\left\|u^{h}\right\|_{1 h}\left\|v^{h}\right\|_{1 h}\left\|w^{h}\right\|_{1 h}$
(2) $\left|c_{1 h}\left(B^{h} ; w^{h}, \Psi^{h}\right)\right| \leq C\left\|\nabla \times \Psi^{h}\right\|_{0}\left\|B^{h}\right\|_{1}\left\|w^{h}\right\|_{1 h}$
(3) $\left|c_{2 h}\left(B^{h} ; v^{h}, \Phi^{h}\right)\right| \leq C\left\|\nabla \times \Phi^{h}\right\|_{0}\left\|B^{h}\right\|_{1}\left\|v^{h}\right\|_{1 h}$.

Proof. The first result is wellknown $[30,37,38]$. To prove the second result, we need the imbedding properties $H_{n}^{1}(\Omega)^{3} \hookrightarrow H^{1}(\Omega)^{3} \hookrightarrow L^{4}(\Omega)^{3}$ and the discrete imbedding inequality showed in $[30,32]$ :

$$
\left\|v^{h}\right\|_{0,2 k, \Omega} \leq C\left\|v^{h}\right\|_{1 h^{\prime}} \quad \forall v^{h} \in X_{1 h}, k=1,2 .
$$

Thus,

$$
\begin{aligned}
\left|c_{1 h}\left(B^{h} ; w^{h}, \Psi^{h}\right)\right| & \leq \sum_{K \in \Gamma^{h}} \int_{K} \frac{1}{R_{m}}\left|\left(\nabla \times \Psi^{h}\right) \times B^{h} \cdot w^{h}\right| d \mathbf{x} \\
& \leq \frac{1}{R_{m}}\left\|\left(\nabla \times \Psi^{h}\right)\right\|_{0}\left\|B^{h}\right\|_{0,4}\left\|w^{h}\right\|_{0,4} \\
& \leq C\left\|\left(\nabla \times \Psi^{h}\right)\right\|_{0}\left\|B^{h}\right\|_{1}\left\|w^{h}\right\|_{1 h^{\prime}}
\end{aligned}
$$

the assertion for $c_{1 h}$ is proved. The proof for $c_{2 h}$ is analogous.

Lemma 4.4. Let $\left(u^{h}, B^{h}\right),\left(v^{h}, \Psi^{h}\right)$, and $\left(w^{h}, \Phi^{h}\right) \in W_{h}$; then the following results hold:

(1) $\left|a_{1 h}\left(\left(u^{h}, B^{h}\right),\left(v^{h}, \Psi^{h}\right),\left(w^{h}, \Phi^{h}\right)\right)\right| \leq C_{c} \gamma_{3}\left\|\left(u^{h}, B^{h}\right)\right\|_{h}\left\|\left(v^{h}, \Psi^{h}\right)\right\|_{h}\left\|\left(w^{h}, \Phi^{h}\right)\right\|_{h^{\prime}}$

(2) $\left|a_{0 h}\left(\left(u^{h}, B^{h}\right),\left(u^{h}, B^{h}\right)\right)\right| \geq C_{a} r_{1}\left\|\left(u^{h}, B^{h}\right)\right\|_{h^{\prime}}^{2}$

(3) $\left|a_{0 h}\left(\left(u^{h}, B^{h}\right),\left(v^{h}, \Psi^{h}\right)\right)\right| \leq C \gamma_{2}\left\|\left(u^{h}, B^{h}\right)\right\|_{h}\left\|\left(v^{h}, \Psi^{h}\right)\right\|_{h}$,

where $C_{c}, C_{a}$ are positive constants, independent of $h$.

Proof. Firstly, using the triangle inequality and Lemma 4.3 yields

$$
\begin{aligned}
& \left|a_{1 h}\left(\left(u^{h}, B^{h}\right),\left(v^{h}, \Psi^{h}\right),\left(w^{h}, \Phi^{h}\right)\right)\right| \\
& \quad \leq\left|c_{0 h}\left(u^{h} ; v^{h}, w^{h}\right)\right|+\left|c_{1 h}\left(B^{h} ; w^{h}, \Psi^{h}\right)\right|+\left|c_{2 h}\left(B^{h} ; v^{h}, \Phi^{h}\right)\right| \\
& \quad \leq C_{c} \gamma_{3}\left\|\left(u^{h}, B^{h}\right)\right\|_{h}\left\|\left(v^{h}, \Psi^{h}\right)\right\|_{h}\left\|\left(w^{h}, \Phi^{h}\right)\right\|_{h} .
\end{aligned}
$$

Applying $H_{n}^{1}(\Omega)^{3} \hookrightarrow H^{1}(\Omega)^{3}$ and the following inequality $[9,37,38]$

$$
\|v\|_{0} \leq C\left(\|\nabla \times v\|_{0}+\|\nabla \cdot v\|_{0}\right), \quad \forall v \in H_{n}^{1}(\Omega)^{3}
$$


leads to

$$
\begin{aligned}
& a_{0 h}\left(\left(u^{h}, B^{h}\right),\left(u^{h}, B^{h}\right)\right) \\
& \quad=\sum_{K \in \Gamma^{h}}\left\{M^{-2} \int_{K} \nabla u^{h} \cdot \nabla u^{h}+R_{m}^{-2} \int_{K}\left[\left(\nabla \times B^{h}\right) \cdot\left(\nabla \times B^{h}\right)+\left(\nabla \cdot B^{h}\right)\left(\nabla \cdot B^{h}\right)\right]\right\} d \mathbf{x} \\
& \quad=M^{-2}\left\|\nabla u^{h}\right\|_{0 h}^{2}+R_{m}^{-2}\left(\left\|\nabla \times B^{h}\right\|_{0}^{2}+\left\|\nabla \cdot B^{h}\right\|_{0}^{2}\right) \\
& \quad \geq C_{a} \min \left\{M^{-2}, R_{m}^{-2}\right\}\left(\left\|u^{h}\right\|_{1 h}^{2}+\left\|B^{h}\right\|_{1}^{2}\right) \\
& \quad=C_{a} \gamma_{1}\left\|\left(u^{h}, B^{h}\right)\right\|_{h}^{2} .
\end{aligned}
$$

With the help of Hölder's inequality, we find

$$
\begin{aligned}
& \left|a_{0 h}\left(\left(u^{h}, B^{h}\right),\left(v^{h}, \Psi^{h}\right)\right)\right| \\
& \quad \leq \sum_{K \in \Gamma^{h}}\left\{M^{-2} \int_{K}\left|\nabla u^{h} \cdot \nabla v^{h}\right|+R_{m}^{-2} \int_{K}\left[\left|\left(\nabla \times B^{h}\right) \cdot\left(\nabla \times \Psi^{h}\right)\right|+\left|\left(\nabla \cdot B^{h}\right)\left(\nabla \cdot \Psi^{h}\right)\right|\right]\right\} d \mathbf{x} \\
& \quad \leq\left\{M^{-2}\left\|\nabla u^{h}\right\|_{0 h}\left\|\nabla v^{h}\right\|_{0 h}+C R_{m}^{-2}\left(\left\|\nabla \times B^{h}\right\|_{0}\left\|\nabla \times \Psi^{h}\right\|_{0}+\left\|\nabla \cdot B^{h}\right\|_{0}\left\|\nabla \cdot \Psi^{h}\right\|_{0}\right)\right\} \\
& \quad \leq C \max \left\{M^{-2}, R_{m}^{-2}\right\}\left\|\left(u^{h}, B^{h}\right)\right\|_{h}\left\|\left(v^{h}, \Psi^{h}\right)\right\|_{h} \\
& \quad=C \gamma_{2}\left\|\left(u^{h}, B^{h}\right)\right\|_{h}\left\|\left(v^{h}, \Psi^{h}\right)\right\|_{h} .
\end{aligned}
$$

The proof is completed.

Lemma 4.5. The spaces $X_{1 h}$ and $M_{h}$ satisfy the discrete inf-sup condition [37, 38]; that is, there exists $\beta^{\star}>0$ such that

$$
\inf _{x^{h} \in M_{h}} \sup _{\left(v^{h}, \Psi^{h}\right) \in W_{h}} \frac{b_{h}\left(\left(v^{h}, \Psi^{h}\right), x^{h}\right)}{\left\|\left(v^{h}, \Psi^{h}\right)\right\|_{h}\left\|x^{h}\right\|_{0}} \geq \beta^{\star} .
$$

Proof. On the one hand, by $[37,38]$, there exists a constant $\beta>0$ such that

$$
\inf _{x \in L_{0}^{2}(\Omega)} \sup _{(v, \Psi) \in W(\Omega)} \frac{b((v, \Psi), \chi)}{\|(v, \Psi)\|_{W}\|\chi\|_{0}} \geq \beta
$$


Therefore, by the assumption (E) and (4.22), we obtain

$$
\begin{aligned}
\sup _{\left(v^{h}, \Psi^{h}\right) \in W_{h}} \frac{b_{h}\left(\left(v^{h}, \Psi^{h}\right), x^{h}\right)}{\left\|\left(v^{h}, \Psi^{h}\right)\right\|_{h}} & \geq \sup _{\left(v, \Psi^{h}\right) \in H_{0}^{1}(\Omega)^{3} \times X_{2 h}} \frac{b_{h}\left(\left(\Pi^{1} v, \Psi^{h}\right), x^{h}\right)}{\left\|\left(\Pi^{1} v, \Psi^{h}\right)\right\|_{h}} \\
& =\sup _{\left(v, \Psi^{h}\right) \in H_{0}^{1}(\Omega)^{3} \times X_{2 h}} \frac{b_{h}\left(\left(v, \Psi^{h}\right), x^{h}\right)}{\left\|\left(\Pi^{1} v, \Psi^{h}\right)\right\|_{h}} \\
& \geq \frac{1}{C} \sup _{\left(v, \Psi^{h}\right) \in H_{0}^{1}(\Omega)^{3} \times X_{2 h}} \frac{b\left(\left(v, \Psi^{h}\right), x^{h}\right)}{\left\|\left(v, \Psi^{h}\right)\right\|_{h}} \\
& \geq \beta^{*}\left\|x^{h}\right\|_{0^{\prime}}
\end{aligned}
$$

where $\beta^{\star}=\beta / C>0$. The proof is completed.

From Lemmas 4.4-4.5, we have the following.

Theorem 4.6. For $f \in H^{-1}(\Omega)^{3}$, Problem $\left(\mathbf{I}_{2}\right)$ has at least one solution $\left(\left(u^{h}, B^{h}\right), p^{h}\right) \in W_{h} \times$ $M_{h}$ satisfying the stability bound $\left\|\left(u^{h}, B^{h}\right)\right\|_{h} \leq\left(C_{a} \gamma_{1}\right)^{-1}\|f\|_{h}$. Moreover, Problem $\left(\mathbf{I}_{2}\right)$ has a unique solution provided that $C_{c} \gamma_{3}\left(C_{a} \gamma_{1}\right)^{-2}\|f\|_{h}<1$.

\section{The Convergence Analysis}

In this section, we will state the main results of this paper, that is, the error estimates for the velocity and the magnetic fields in $H^{1}$-norm.

Theorem 5.1. Assume that

$$
\frac{C_{c} r_{3}\|f\|_{-1}}{C_{a} C_{1} r_{1}^{2}}<\frac{1}{2}
$$

Let $((u, B), p) \in W(\Omega) \times L_{0}^{2}(\Omega)$ and $\left(\left(u^{h}, B^{h}\right), p^{h}\right) \in W_{h} \times M_{h}$ be the solutions of Problems $\left(\mathbf{I}_{1}\right)$ and $\left(\mathbf{I}_{2}\right)$, respectively. Then there hold

(1)

$$
\begin{aligned}
& \left\|(u, B)-\left(u^{h}, B^{h}\right)\right\|_{h} \\
& \quad \leq C\left\{\inf _{\left(v^{h}, \Psi^{h}\right) \in W_{h}}\left\|(u, B)-\left(v^{h}, \Psi^{h}\right)\right\|_{h}+\inf _{s^{h} \in M_{h}}\left\|p-s^{h}\right\|_{0}+\sup _{\left(v^{h}, \Psi^{h}\right) \in Z_{h} \times X_{2 h}} \frac{\left|E\left(\left(v^{h}, \Psi^{h}\right)\right)\right|}{\left\|\left(v^{h}, \Psi^{h}\right)\right\|_{h}}\right\},
\end{aligned}
$$

(2)

$$
\begin{aligned}
& \left\|p-p^{h}\right\|_{0} \\
& \quad \leq C\left\{\inf _{\left(v^{h}, \Psi^{h}\right) \in W_{h}}\left\|(u, B)-\left(v^{h}, \Psi^{h}\right)\right\|_{h}+\inf _{s^{h} \in M_{h}}\left\|p-s^{h}\right\|_{0}+\sup _{\left(v^{h}, \Psi^{h}\right) \in W_{h}} \frac{\left|E\left(\left(v^{h}, \Psi^{h}\right)\right)\right|}{\left\|\left(v^{h}, \Psi^{h}\right)\right\|_{h}}\right\},
\end{aligned}
$$


Journal of Applied Mathematics

where

$$
E\left(\left(v^{h}, \Psi^{h}\right)\right)=\sum_{K \in \Gamma^{h}} \int_{\partial K}\left[M^{-2} \frac{\partial u}{\partial n} v^{h}-p v^{h} \cdot n-(2 N)^{-1}(u \cdot n)\left(u \cdot v^{h}\right)\right] d s .
$$

Proof. We proceed in two steps.

Step 1. For $\left(v^{h}, \Psi^{h}\right) \in W_{h}$, by Green's formula, we have

$$
\begin{aligned}
& a_{0 h}\left((u, B),\left(v^{h}, \Psi^{h}\right)\right)+a_{1 h}\left((u, B),(u, B),\left(v^{h}, \Psi^{h}\right)\right)+b_{h}\left(\left(v^{h}, \Psi^{h}\right), p\right)-F\left(\left(v^{h}, \Psi^{h}\right)\right) \\
& =\sum_{K \in \Gamma^{h}}\left\{\int_{K} M^{-2} \nabla u \cdot \nabla v^{h} d \mathbf{x}+R_{m}^{-2} \int_{K}(\nabla \times B) \cdot\left(\nabla \times \Psi^{h}\right) d \mathbf{x}\right. \\
& +(2 N)^{-1} \int_{K}\left(u \cdot \nabla u \cdot v^{h}-u \cdot \nabla v^{h} \cdot u\right) d \mathbf{x} \\
& -R_{m}^{-1} \int_{K}\left[(\nabla \times B) \times B \cdot v^{h}-\left(\nabla \times \Psi^{h}\right) \times B \cdot u\right] d \mathbf{x} \\
& \left.-\int_{K} p \cdot \nabla v^{h} d \mathbf{x}-\int_{K} f v^{h} d \mathbf{x}\right\} \\
& =\sum_{K \in \Gamma^{h}}\left\{\int_{K}-M^{-2} \Delta u \cdot v^{h} d \mathbf{x}+\int_{\partial K} M^{-2} \frac{\partial u}{\partial n} v^{h} d s\right. \\
& +\int_{K} R_{m}^{-2} \nabla \times(\nabla \times B) \cdot \Psi^{h} d \mathbf{x}+\int_{\partial K} R_{m}^{-2}(\nabla \times B \times n) \cdot \Psi^{h} d s \\
& +\int_{K} N^{-1} u \cdot \nabla u \cdot v^{h} d \mathbf{x}-\int_{\partial K}(2 N)^{-1}(u \cdot n)\left(u \cdot v^{h}\right) d s \\
& -R_{m}^{-1} \int_{K}(\nabla \times B) \times B \cdot v^{h} d \mathbf{x}-R_{m}^{-1} \int_{K}(\nabla \times u \times B) \cdot \Psi^{h} d \mathbf{x} \\
& \left.+\int_{\partial K}(u \times B \times n) \cdot \Psi^{h} d s+\int_{K} \nabla p \cdot v^{h} d \mathbf{x}-\int_{\partial K} p v^{h} \cdot n d s-\int_{K} f v^{h} d \mathbf{x}\right\} \\
& =\sum_{K \in \Gamma^{h}}\left\{\int_{K}\left(-M^{-2} \Delta u+N^{-1} u \cdot \nabla u+\nabla p-R_{m}^{-1}(\nabla \times B) \times B-f\right) \cdot v^{h}\right. \\
& +R_{m}^{-1} \int_{K}\left[R_{m}^{-1} \nabla \times(\nabla \times B)-\nabla \times(u \times B)\right] \cdot \Psi^{h} d \mathbf{x} \\
& \left.+\int_{\partial K}\left[M^{-2} \frac{\partial u}{\partial n} v^{h}-(2 N)^{-1}(u \cdot n)\left(u \cdot v^{h}\right)-p v^{h} \cdot n\right] d s\right\} \\
& =E\left(\left(v^{h}, \Psi^{h}\right)\right) \text {. }
\end{aligned}
$$

Thus,

$$
\begin{aligned}
& a_{0 h}\left((u, B),\left(v^{h}, \Psi^{h}\right)\right)+a_{1 h}\left((u, B),(u, B),\left(v^{h}, \Psi^{h}\right)\right)+b_{h}\left(\left(v^{h}, \Psi^{h}\right), p\right) \\
& \quad=F\left(\left(v^{h}, \Psi^{h}\right)\right)+E\left(\left(v^{h}, \Psi^{h}\right)\right) .
\end{aligned}
$$


Here, we have used the following equality:

$$
\int_{\Omega}(\nabla \times \Phi) \cdot \Psi d \mathbf{x}=-\int_{\partial \Omega}(\Phi \times n) \cdot \Psi d s+\int_{\Omega} \Phi \cdot(\nabla \times \Psi) d \mathbf{x}
$$

On the other hand, we have from (4.8)

$$
\begin{aligned}
& a_{0 h}\left(\left(u^{h}, B^{h}\right),\left(v^{h}, \Psi^{h}\right)\right)+a_{1 h}\left(\left(u^{h}, B^{h}\right),\left(u^{h}, B^{h}\right),\left(v^{h}, \Psi^{h}\right)\right)+b_{h}\left(\left(v^{h}, \Psi^{h}\right), p^{h}\right) \\
& \quad=F\left(\left(v^{h}, \Psi^{h}\right)\right) .
\end{aligned}
$$

Subtraction of (4.8) from (5.6) yields

$$
\begin{aligned}
& a_{0 h}\left((u, B)-\left(u^{h}, B^{h}\right),\left(v^{h}, \Psi^{h}\right)\right)+a_{1 h}\left((u, B)-\left(u^{h}, B^{h}\right),(u, B),\left(v^{h}, \Psi^{h}\right)\right) \\
& \quad+a_{1 h}\left(\left(u^{h}, B^{h}\right),(u, B)-\left(u^{h}, B^{h}\right),\left(v^{h}, \Psi^{h}\right)\right) \\
& \quad+b_{h}\left(\left(v^{h}, \Psi^{h}\right), p-p^{h}\right)=E\left(\left(v^{h}, \Psi^{h}\right)\right) .
\end{aligned}
$$

Let $\left(w^{h}, \Phi^{h}\right)$ be an arbitrary element of $Z_{h} \times X_{2 h}$, that is:

$$
b_{h}\left(\left(w^{h}, \Phi^{h}\right), x^{h}\right)=0, \quad \forall x^{h} \in M_{h} .
$$

Then,

$$
b_{h}\left(\left(u^{h}-w^{h}, B^{h}-\Phi^{h}\right), x^{h}\right)=b_{h}\left(\left(u^{h}, B^{h}\right), x^{h}\right)-b_{h}\left(\left(w^{h}, \Phi^{h}\right), x^{h}\right)=0 .
$$

For all $\left(v^{h}, \Psi^{h}\right) \in W_{h}, s^{h} \in M_{h}$, by virtue of $\left(u^{h}-w^{h}, B^{h}-\Phi^{h}\right) \in Z_{h} \times X_{2 h}$ and (5.9), we get

$$
\begin{aligned}
& a_{0 h}\left(\left(w^{h}, \Phi^{h}\right)-\left(u^{h}, B^{h}\right),\left(v^{h}, \Psi^{h}\right)\right)+a_{1 h}\left(\left(w^{h}, \Phi^{h}\right)-\left(u^{h}, B^{h}\right),(u, B),\left(v^{h}, \Psi^{h}\right)\right) \\
& +a_{1 h}\left(\left(u^{h}, B^{h}\right),\left(w^{h}, \Phi^{h}\right)-\left(u^{h}, B^{h}\right),\left(v^{h}, \Psi^{h}\right)\right)+b_{h}\left(\left(v^{h}, \Psi^{h}\right), s^{h}-p^{h}\right) \\
& =a_{0 h}\left(\left(w^{h}, \Phi^{h}\right)-(u, B),\left(v^{h}, \Psi^{h}\right)\right)+a_{1 h}\left(\left(w^{h}, \Phi^{h}\right)-(u, B),(u, B),\left(v^{h}, \Psi^{h}\right)\right) \\
& \quad+a_{1 h}\left(\left(u^{h}, B^{h}\right),\left(w^{h}, \Phi^{h}\right)-(u, B),\left(v^{h}, \Psi^{h}\right)\right)+b_{h}\left(\left(v^{h}, \Psi^{h}\right), s^{h}-p\right) \\
& +E\left(\left(v^{h}, \Psi^{h}\right)\right) .
\end{aligned}
$$

Notice that

$$
\begin{gathered}
a_{1 h}\left(\left(u^{h}, B^{h}\right),\left(w^{h}, \Phi^{h}\right)-\left(u^{h}, B^{h}\right),\left(w^{h}, \Phi^{h}\right)-\left(u^{h}, B^{h}\right)\right)=0, \\
b_{h}\left(\left(u^{h}-w^{h}, B^{h}-\Phi^{h}\right), s^{h}-p^{h}\right)=0 .
\end{gathered}
$$


Let $\left(v^{h}, \Psi^{h}\right)=\left(w^{h}, \Phi^{h}\right)-\left(u^{h}, B^{h}\right)$, and by (5.12), we obtain

$$
\begin{aligned}
& a_{0 h}\left(\left(w^{h}, \Phi^{h}\right)-\left(u^{h}, B^{h}\right),\left(w^{h}, \Phi^{h}\right)-\left(u^{h}, B^{h}\right)\right) \\
& +a_{1 h}\left(\left(w^{h}, \Phi^{h}\right)-\left(u^{h}, B^{h}\right),(u, B),\left(w^{h}, \Phi^{h}\right)-\left(u^{h}, B^{h}\right)\right) \\
& \quad=a_{0 h}\left(\left(w^{h}, \Phi^{h}\right)-(u, B),\left(w^{h}, \Phi^{h}\right)-\left(u^{h}, B^{h}\right)\right) \\
& \quad+a_{1 h}\left(\left(w^{h}, \Phi^{h}\right)-(u, B),(u, B),\left(w^{h}, \Phi^{h}\right)-\left(u^{h}, B^{h}\right)\right) \\
& \quad+a_{1 h}\left(\left(u^{h}, B^{h}\right),\left(w^{h}, \Phi^{h}\right)-(u, B),\left(w^{h}, \Phi^{h}\right)-\left(u^{h}, B^{h}\right)\right) \\
& \quad+b_{h}\left(\left(w^{h}, \Phi^{h}\right)-\left(u^{h}, B^{h}\right), s^{h}-p\right)+E\left(\left(w^{h}, \Phi^{h}\right)-\left(u^{h}, B^{h}\right)\right) .
\end{aligned}
$$

Using the continuity properties of $a_{0 h}, a_{1 h}$ and the stability bounds for $\|(u, B)\|_{W}$ and $\left\|\left(u^{h}, B^{h}\right)\right\|_{h}$ in Theorems 2.1 and 4.6, respectively, the right-hand side of (5.14) can be bounded by

$$
\begin{aligned}
\text { r.h.s. } \leq & \left\|\left(w^{h}, \Phi^{h}\right)-\left(u^{h}, B^{h}\right)\right\|_{h} \\
& \times\left[C \gamma_{2}\left\|\left(w^{h}, \Phi^{h}\right)-(u, B)\right\|_{h}+C_{c}\left\|\left(w^{h}, \Phi^{h}\right)-(u, B)\right\|_{h}\|(u, B)\|_{W}\right. \\
& \left.\quad+C_{c}\left\|\left(w^{h}, \Phi^{h}\right)-(u, B)\right\|_{h}\left\|\left(u^{h}, B^{h}\right)\right\|_{h}+C\left\|s^{h}-p\right\|_{0}+\frac{E\left(\left(w^{h}, \Phi^{h}\right)-\left(u^{h}, B^{h}\right)\right)}{\left\|\left(w^{h}, \Phi^{h}\right)-\left(u^{h}, B^{h}\right)\right\|_{h}}\right] \\
\leq C & \left\|\left(w^{h}, \Phi^{h}\right)-\left(u^{h}, B^{h}\right)\right\|_{h}\left[\left\|\left(w^{h}, \Phi^{h}\right)-(u, B)\right\|_{h}+\left\|s^{h}-p\right\|_{0}+\frac{E\left(\left(w^{h}, \Phi^{h}\right)-\left(u^{h}, B^{h}\right)\right)}{\left\|\left(w^{h}, \Phi^{h}\right)-\left(u^{h}, B^{h}\right)\right\|_{h}}\right] .
\end{aligned}
$$

Next, the coercivity property of the form $a_{0 h}$, continuity of $a_{1 h}$ in Lemma 4.4 , stability bound for $\|(u, B)\|_{W}$ in Theorem 2.1, and the assumption $C_{c} \gamma_{3}\|f\|_{-1} / C_{a} C_{1} \gamma_{1}^{2}<1 / 2$ allow us to bound the left-hand side of (5.14) as

$$
\begin{aligned}
\text { 1.h.s. } & \geq C_{a} \gamma_{1}\left\|\left(w^{h}, \Phi^{h}\right)-\left(u^{h}, B^{h}\right)\right\|_{h}^{2}-C_{c} r_{3}\left\|\left(w^{h}, \Phi^{h}\right)-\left(u^{h}, B^{h}\right)\right\|_{h}^{2}\|(u, B)\|_{W} \\
& \geq \frac{1}{2} C_{a} \gamma_{1}\left\|\left(w^{h}, \Phi^{h}\right)-\left(u^{h}, B^{h}\right)\right\|_{h}^{2} .
\end{aligned}
$$

Combining these bounds, we have

$$
\left\|\left(w^{h}, \Phi^{h}\right)-\left(u^{h}, B^{h}\right)\right\|_{h} \leq C\left[\left\|\left(w^{h}, \Phi^{h}\right)-(u, B)\right\|_{h}+\left\|s^{h}-p\right\|_{0}+\frac{E\left(\left(w^{h}, \Phi^{h}\right)-\left(u^{h}, B^{h}\right)\right)}{\left\|\left(w^{h}, \Phi^{h}\right)-\left(u^{h}, B^{h}\right)\right\|_{h}}\right] .
$$


Then, applying the triangle inequality, we get

$$
\left\|(u, B)-\left(u^{h}, B^{h}\right)\right\|_{h} \leq C\left[\left\|\left(w^{h}, \Phi^{h}\right)-(u, B)\right\|_{h}+\left\|s^{h}-p\right\|_{0}+\frac{E\left(\left(w^{h}, \Phi^{h}\right)-\left(u^{h}, B^{h}\right)\right)}{\left\|\left(w^{h}, \Phi^{h}\right)-\left(u^{h}, B^{h}\right)\right\|_{h}}\right] .
$$

Now, for $\left(w^{h}, \Phi^{h}\right) \in Z_{h} \times X_{2 h}, s^{h} \in M_{h}$, taking the infimum of (5.18) yields

$$
\begin{aligned}
& \left\|(u, B)-\left(u^{h}, B^{h}\right)\right\|_{h} \\
& \quad \leq C\left[\inf _{\left(w^{h}, \Phi^{h}\right) \in Z_{h} \times X_{2 h}}\left\|\left(w^{h}, \Phi^{h}\right)-(u, B)\right\|_{h}+\inf _{s^{h} \in M_{h}}\left\|s^{h}-p\right\|_{0}+\sup _{\left(v^{h}, \Psi^{h}\right) \in Z_{h} \times X_{2 h}} \frac{E\left(\left(v^{h}, \Psi^{h}\right)\right)}{\left\|\left(v^{h}, \Psi^{h}\right)\right\|_{h}}\right] .
\end{aligned}
$$

With the argument as [37], we know that

$$
\inf _{\left(w^{h}, \Phi^{h}\right) \in Z_{h} \times X_{2 h}}\left\|\left(w^{h}, \Phi^{h}\right)-(u, B)\right\|_{h} \leq C_{\left(v^{h}, \Psi^{h}\right) \in W_{h}}\left\|\left(w^{h}, \Phi^{h}\right)-(u, B)\right\|_{h} .
$$

Substituting (5.20) into (5.19) implies (5.2).

Step 2. For $\left(v^{h}, \Psi^{h}\right) \in W_{h}, s^{h} \in M_{h}$, we have from (5.9) that

$$
\begin{aligned}
b_{h}\left(\left(v^{h}, \Psi^{h}\right), s^{h}-p^{h}\right)= & b_{h}\left(\left(v^{h}, \Psi^{h}\right), s^{h}-p\right)+b_{h}\left(\left(v^{h}, \Psi^{h}\right), p-p^{h}\right) \\
= & b_{h}\left(\left(v^{h}, \Psi^{h}\right), s^{h}-p\right)-a_{0 h}\left((u, B)-\left(u^{h}, B^{h}\right),\left(v^{h}, \Psi^{h}\right)\right) \\
& -a_{1 h}\left((u, B)-\left(u^{h}, B^{h}\right),(u, B),\left(v^{h}, \Psi^{h}\right)\right) \\
& -a_{1 h}\left(\left(u^{h}, B^{h}\right),(u, B)-\left(u^{h}, B^{h}\right),\left(v^{h}, \Psi^{h}\right)\right) \\
& +E\left(\left(v^{h}, \Psi^{h}\right)\right) .
\end{aligned}
$$

Using the continuity properties of $a_{0 h}$ and $a_{1 h}$ and the discrete inf-sup condition (4.21) of Lemma 4.5 , it follows that

$$
\begin{aligned}
& \left\|s^{h}-p^{h}\right\|_{0} \\
& \leq \frac{1}{\beta^{\star}}\left\{C\left\|s^{h}-p\right\|_{0}+\left[C \gamma_{2}+C_{c}\left(\|(u, B)\|_{W}+\left\|\left(u^{h}, B^{h}\right)\right\|_{h}\right)\right]\left\|(u, B)-\left(u^{h}, B^{h}\right)\right\|_{h}+\frac{E\left(\left(v^{h}, \Psi^{h}\right)\right)}{\left\|\left(v^{h}, \Psi^{h}\right)\right\|_{h}}\right\} .
\end{aligned}
$$

Then, with the help of the triangle inequality and (5.2), we complete the proof. 
Theorem 5.2. Let $u \in\left(H_{0}^{1}(\Omega)^{3} \cap H^{2}(\Omega)^{3}\right), B \in\left(H^{2}(\Omega)^{3} \cap H_{n}^{1}(\Omega)^{3}\right), p \in\left(L_{0}^{2}(\Omega) \cap H^{1}(\Omega)\right)$, and $\left(\left(u^{h}, B^{h}\right), p^{h}\right) \in W_{h} \times M_{h}$ be the solutions of Problems $\left(\mathbf{I}_{1}\right)$ and $\left(\mathbf{I}_{2}\right)$, respectively. Then there holds

$$
\left\|(u, B)-\left(u^{h}, B^{h}\right)\right\|_{h}+\left\|p-p^{h}\right\|_{0} \leq C h\left(|u|_{2}+\|B\|_{2}+\|p\|_{1}\right) \text {. }
$$

Proof. On the one hand, the interpolation theory gives

$$
\begin{gathered}
\inf _{v^{h} \in X_{1 h}}\left\|u-v^{h}\right\|_{1 h}^{2} \leq\left\|u-\Pi^{1} u\right\|_{1 h}^{2} \leq C h^{2}|u|_{2}^{2}, \\
\inf _{\Psi^{h} \in X_{2 h}}\left\|B-\Psi^{h}\right\|_{1}^{2} \leq C h^{2}\|B\|_{2}^{2} .
\end{gathered}
$$

Therefore, by (5.24), we obtain

$$
\inf _{\left(v^{h}, \Psi^{h}\right) \in W_{h}}\left\|(u, B)-\left(v^{h}, \Psi^{h}\right)\right\|_{h} \leq C h\left(|u|_{2}+\|B\|_{2}\right) .
$$

At the same time, for $p \in L_{0}^{2}(\Omega)$, we define the interpolation $R_{0}^{h} p \in M_{h}$ on each element $K$ as

$$
\int_{K}\left(p-R_{0}^{h} p\right) d \mathbf{x}=0
$$

Then there holds

$$
\inf _{s^{h} \in M_{h}}\left\|p-s^{h}\right\|_{0} \leq\left\|p-R_{0}^{h} p\right\|_{0} \leq C h\|p\|_{1} .
$$

On the other hand, by the similar techniques to [25-27, 29, 30, 32], we have

$$
\left|E\left(\left(v^{h}, \Psi^{h}\right)\right)\right| \leq C h\left(|u|_{2}+\|p\|_{1}\right)\left\|\left(v^{h}, \Psi^{h}\right)\right\|_{h} .
$$

Substituting (5.24)-(5.28) into (5.2) and (5.3) yields the desired result.

Next, we will establish the error estimates in $L^{2}$-norm for the velocity and the magnetic fields by use of the duality argument introduced in [46].

We consider the following dual problem. Find $(w, \Phi)$ and $s$ such that.

$$
\begin{aligned}
& -M^{-2} \Delta w+N^{-1}[w \cdot \nabla u-u \cdot \nabla w]+\nabla s+R_{m}^{-1}(\nabla \times \Phi) \times B=u-u^{h}, \quad \text { in } \Omega, \\
& R_{m}^{-2}[\nabla \times(\nabla \times \Phi)-\nabla(\nabla \cdot \Phi)]+R_{m}^{-1}[(\nabla \times B) \times w-(\nabla \times \Phi) \times u-\nabla \times(B \times w)] \\
& \quad=B-B^{h}, \quad \text { in } \Omega, \\
& \nabla \cdot w=0, \quad \text { in } \Omega, \\
& w=0, \quad \text { on } \partial \Omega, \\
& B \cdot n=0, \quad R_{m}^{-1}(\nabla \times \Phi) \times n+w \times B \times n=0, \quad \text { on } \partial \Omega .
\end{aligned}
$$

The variational formulation of (5.29) is written as follows. 
Problem $\left(\mathbf{I}_{3}\right)$. Find $(w, \Phi) \in W(\Omega)$ and $s \in L_{0}^{2}(\Omega)$ such that for all $(v, \Psi) \in W(\Omega), \psi \in L_{0}^{2}(\Omega)$

$$
\begin{aligned}
& a_{0}((v, \Psi),(w, \Phi))+a_{1}((u, B),(v, \Psi),(w, \Phi))+a_{1}((v, \Psi),(u, B),(w, \Phi))+b((v, \Psi), s) \\
& \quad=\left((u, B)-\left(u^{h}, B^{h}\right),(v, \Psi)\right), \\
& b((w, \Phi), \psi)=0 .
\end{aligned}
$$

Under the same hypotheses as Theorem 2.1, we may easily know that Problem $\left(\mathbf{I}_{3}\right)$ has a unique solution $((w, \Phi), s) \in W(\Omega) \times L_{0}^{2}(\Omega)$.

We require that (5.29) be $H^{2}$-regular, that is:

$$
\|(w, \Phi)\|_{2}+\|s\|_{1} \leq C\left\|(u, B)-\left(u^{h}, B^{h}\right)\right\|_{0} .
$$

Let $\left(\left(w^{h}, \Phi^{h}\right), s^{h}\right) \in W_{h} \times M_{h}$ satisfy

$$
\left\|(w, \Phi)-\left(w^{h}, \Phi^{h}\right)\right\|_{h}+\left\|s-s^{h}\right\|_{0} \leq C h\left(|w|_{2}+\|\Phi\|_{2}+\|s\|_{1}\right) .
$$

Theorem 5.3. Under the hypothesis of Theorem 5.2, let $((w, \Phi), s)$ be the solution of Problem $\left(\mathbf{I}_{3}\right)$, and assume that (5.31) holds. Then we have

$$
\left\|(u, B)-\left(u^{h}, B^{h}\right)\right\|_{0} \leq C h^{2}\left(|u|_{2}+\|B\|_{2}+\|p\|_{1}\right) .
$$

Proof. By (5.31) and (5.32), we deduce that

$$
\left\|(w, \Phi)-\left(w^{h}, \Phi^{h}\right)\right\|_{h}+\left\|s-s^{h}\right\|_{0} \leq C h\left\|(u, B)-\left(u^{h}, B^{h}\right)\right\|_{0} .
$$

Multiplying $\left(u-u^{h}\right)$ and $\left(B-B^{h}\right)$ both sides of the first and the second equation of (5.29), respectively, and integrating by parts on each element, we see that

$$
\begin{aligned}
\left\|(u, B)-\left(u^{h}, B^{h}\right)\right\|_{0}^{2}= & a_{0 h}\left((u, B)-\left(u^{h}, B^{h}\right),(w, \Phi)\right)+a_{1 h}\left((u, B),(u, B)-\left(u^{h}, B^{h}\right),(w, \Phi)\right) \\
& +a_{1 h}\left((u, B)-\left(u^{h}, B^{h}\right),(u, B),(w, \Phi)\right)+b_{h}\left((u, B)-\left(u^{h}, B^{h}\right), s\right) \\
& -(2 N)^{-1} \sum_{K \in \Gamma^{h}} \int_{K} \operatorname{div}\left(u-u^{h}\right)(u \cdot w) d \mathbf{x}+F\left(\left(u-u^{h}\right)\right),
\end{aligned}
$$


where

$$
\begin{aligned}
F\left(\left(u-u^{h}\right)\right)= & -M^{-2} \sum_{K \in \Gamma^{h}} \int_{\partial K} \frac{\partial w}{\partial n}\left(u-u^{h}\right) d s-(2 N)^{-1} \sum_{K \in \Gamma^{h}} \int_{\partial K}(u \cdot n)\left(w \cdot\left(u-u^{h}\right)\right) d s \\
& +(2 N)^{-1} \sum_{K \in \Gamma^{h}} \int_{\partial K}\left(\left(u-u^{h}\right) \cdot n\right)(u \cdot w) d s+\sum_{K \in \Gamma^{h}} \int_{\partial K} s\left(u-u^{h}\right) \cdot n d s .
\end{aligned}
$$

Subtraction of (5.9) yields

$$
\begin{aligned}
& a_{0 h}\left((u, B)-\left(u^{h}, B^{h}\right),\left(v^{h}, \Psi^{h}\right)\right)+a_{1 h}\left((u, B)-\left(u^{h}, B^{h}\right),(u, B),\left(v^{h}, \Psi^{h}\right)\right) \\
& +a_{1 h}\left(\left(u^{h}, B^{h}\right),(u, B)-\left(u^{h}, B^{h}\right),\left(v^{h}, \Psi^{h}\right)\right)+b_{h}\left(\left(v^{h}, \Psi^{h}\right), p-p^{h}\right) \\
& \quad=E\left(\left(v^{h}, \Psi^{h}\right)\right) .
\end{aligned}
$$

Note that

$$
b_{h}\left((u, B)-\left(u^{h}, B^{h}\right), \phi^{h}\right)=0, \quad \forall \phi^{h} \in M_{h} .
$$

Now, setting $\psi=p-p^{h}$ in Problem $\left(\mathbf{I}_{3}\right)$, we have

$$
b\left((w, \Phi), p-p^{h}\right)=0 .
$$

From (5.35)-(5.39), we get

$$
\begin{aligned}
\|(u, B) & -\left(u^{h}, B^{h}\right) \|_{0}^{2} \\
= & a_{0 h}\left((u, B)-\left(u^{h}, B^{h}\right),(w, \Phi)-\left(v^{h}, \Psi^{h}\right)\right)+b_{h}\left((u, B)-\left(u^{h}, B^{h}\right), s-\phi^{h}\right) \\
& +b_{h}\left((w, \Phi)-\left(v^{h}, \Psi^{h}\right), p-p^{h}\right)+A_{1}+A_{2}+A_{3},
\end{aligned}
$$

where

$$
\begin{aligned}
A_{1}= & a_{1 h}\left(\left(u^{h}, B^{h}\right),(u, B)-\left(u^{h}, B^{h}\right),(w, \Phi)-\left(v^{h}, \Psi^{h}\right)\right) \\
& +a_{1 h}\left((u, B)-\left(u^{h}, B^{h}\right),(u, B),(w, \Phi)-\left(v^{h}, \Psi^{h}\right)\right) \\
& +a_{1 h}\left((u, B)-\left(u^{h}, B^{h}\right),(u, B)-\left(u^{h}, B^{h}\right),(w, \Phi)\right), \\
A_{2}= & F\left(\left(u-u^{h}\right)\right)+E\left(\left(v^{h}, \Psi^{h}\right)\right), \\
A_{3}= & -\frac{1}{2 N} \sum_{K \in \Gamma^{h}} \int_{K} \operatorname{div}\left(u-u^{h}\right)(u \cdot w) d \mathbf{x}
\end{aligned}
$$


By (2.8), Lemma 4.4, and Theorem 4.6, we find

$$
A_{1} \leq C\left(\left\|(u, B)-\left(u^{h}, B^{h}\right)\right\|_{h}\left\|(w, \Phi)-\left(v^{h}, \Psi^{h}\right)\right\|_{h}+\left\|(u, B)-\left(u^{h}, B^{h}\right)\right\|_{h}^{2}\|(w, \Phi)\|_{W}\right) .
$$

From [45], we know

$$
F\left(\left(u-u^{h}\right)\right) \leq C h\left(|w|_{2}+\|s\|_{1}\right)\left\|u-u^{h}\right\|_{1 h}
$$

By virtue of $u, w \in H^{2}(\Omega)^{3} \hookrightarrow C^{0}(\Omega)^{2}$, we obtain

$$
\begin{aligned}
& E\left(\left(v^{h}, \Psi^{h}\right)\right) \\
& \quad=\sum_{K \in \Gamma^{h}} \int_{\partial K}\left[M^{-2} \frac{\partial u}{\partial n}\left(v^{h}-w\right)-p\left(v^{h}-w\right) \cdot n-(2 N)^{-1}(u \cdot n)\left(u \cdot\left(v^{h}-w\right)\right)\right] d s \\
& \quad \leq C h\left(|u|_{2}+\|p\|_{1}\right)\left\|v^{h}-w\right\|_{1 h} .
\end{aligned}
$$

Let $a_{K}$ be a constant such that

$$
\left\|u \cdot w-a_{K}\right\|_{0, K} \leq C h\|u \cdot w\|_{1, K} \leq C h\|u\|_{1, K}\|w\|_{2, K} \cdot
$$

Since $\operatorname{div} u=0, b_{h}\left(\left(u^{h}, B^{h}\right), q\right)=0, \forall q \in M_{h}$ and (5.45), we obtain

$$
\begin{aligned}
\left|A_{3}\right| & =\left|-\frac{1}{2 N} \sum_{K \in \Gamma^{h}} \int_{K} \operatorname{div}\left(u-u^{h}\right)\left(u \cdot w-a_{K}\right) d \mathbf{x}\right| \\
& \leq C h\left\|u-u^{h}\right\|_{1 h}\|u\|_{1}\|w\|_{2} .
\end{aligned}
$$

Thus, by (5.31) and the approximation theory, there hold

$$
\begin{gathered}
\inf _{\left(v^{h}, \Psi^{h}\right) \in W_{h}}\left\|(w, \Phi)-\left(v^{h}, \Psi^{h}\right)\right\|_{h} \leq C h\|(w, \Phi)\|_{2} \leq C h\left\|(u, B)-\left(u^{h}, B^{h}\right)\right\|_{0^{\prime}} \\
\inf _{v^{h} \in X_{2 h}}\left\|w-v^{h}\right\|_{1 h} \leq C h\|w\|_{2} \leq C h\left\|(u, B)-\left(u^{h}, B^{h}\right)\right\|_{0^{\prime}} \\
\inf _{\phi^{h} \in M_{h}}\left\|s-\phi^{h}\right\|_{0} \leq C h\left\|_{s}\right\|_{1} \leq C h\left\|(u, B)-\left(u^{h}, B^{h}\right)\right\|_{0^{\prime}} \\
\|(w, \Phi)\|_{W} \leq\|(w, \Phi)\|_{2} \leq C\left\|(u, B)-\left(u^{h}, B^{h}\right)\right\|_{0^{\prime}} \\
\left|b_{h}\left((u, B)-\left(u^{h}, B^{h}\right), s-\phi^{h}\right)\right| \leq C\left\|(u, B)-\left(u^{h}, B^{h}\right)\right\|_{h}\left\|s-\phi^{h}\right\|_{0^{\prime}} \\
\left|b_{h}\left((w, \Phi)-\left(v^{h}, \Psi^{h}\right), p-p^{h}\right)\right| \leq C\left\|(u, B)-\left(u^{h}, B^{h}\right)\right\|_{h}\left\|s-\phi^{h}\right\|_{0}
\end{gathered}
$$


Combining these inequalities and using Lemma 4.4 and the results from (5.39) to (5.46) yields the desired result.

Remark 5.4. The results obtained in this paper are also valid to the MHD equations with the following boundary conditions $u=0, n \times B=0,(\nabla \times B) \cdot n=0$ on $\partial \Omega$ when $u \in H_{0}^{1}(\Omega)^{3}, B \in$ $H=\left\{B \in H^{1}(\Omega)^{3} ;\left.(B \times n)\right|_{\partial \Omega}=0\right\}$.

\section{Acknowledgments}

The research are supported by the National Natural Science Foundation of China (no. 10671184; no. 10971203), the National Science Foundation for Young Scientists of China (no. 11101384) and the Foundation and Advanced Technology Research Program of Henan Province, China (no. 122300410208).

\section{References}

[1] J. A. Shercliff, "Steady motion of conducting fluids in pipes under transverse magnetic fields," Proceedings of the Cambridge Philosophical Society, vol. 49, pp. 136-144, 1953.

[2] J.-F. Gerbeau, C. Le Bris, and T. Lelièvre, Mathematical Methods for the Magnetohydrodynamics of Liquid Metals, Numerical Mathematics and Scientific Computation, Oxford University Press, New York, NY, USA, 2006.

[3] M. Sermange and R. Temam, "Some mathematical questions related to the MHD equations," Communications on Pure and Applied Mathematics, vol. 36, no. 5, pp. 635-664, 1983.

[4] J.-F. Gerbeau and C. Le Bris, "A coupled system arising in magnetohydrodynamics," Applied Mathematics Letters, vol. 12, no. 3, pp. 53-57, 1999.

[5] A. J. Meir and P. G. Schmidt, "Variational methods for stationary MHD flow under natural interface conditions," Nonlinear Analysis: Theory, Methods \& Applications, vol. 26, no. 4, pp. 659-689, 1996.

[6] T. V. S. Sekhar, T. V. R Ravikumar, and H. Kumar, "MHD flow past a sphere at low and moderate Reynolds numbers," Computational Mechanics, vol. 31, pp. 437-444, 2003.

[7] T. W. H. Sheu and R. K. Lin, "Development of a convection-diffusion-reaction magnetohydrodynamic solver on non-staggered grids," International Journal for Numerical Methods in Fluids, vol. 45, no. 11, pp. 1209-1233, 2004.

[8] H. Kumamaru, S. Kodama, H. Hirano, and K. Itoh, "Three-dimensional numerical calculations on liquid-metal magnetohydrodynamic flow in magnetic-field inlet region," Journal of Nuclear Science and Technology, vol. 41, no. 5, pp. 624-631, 2004.

[9] M. D. Gunzburger, A. J. Meir, and J. S. Peterson, "On the existence, uniqueness, and finite element approximation of solutions of the equations of stationary, incompressible magnetohydrodynamics," Mathematics of Computation, vol. 56, no. 194, pp. 523-563, 1991.

[10] M. Wiedmer, "Finite element approximation for equations of magnetohydrodynamics," Mathematics of Computation, vol. 69, no. 229, pp. 83-101, 2000.

[11] F. Armero and J. C. Simo, "Long-term dissipativity of time-stepping algorithms for an abstract evolution equation with applications to the incompressible MHD and Navier-Stokes equations," Computer Methods in Applied Mechanics and Engineering, vol. 131, no. 1-2, pp. 41-90, 1996.

[12] J.-L. Guermond and P. D. Minev, "Mixed finite element approximation of an MHD problem involving conducting and insulating regions: the 2D case," Mathematical Modelling and Numerical Analysis, vol. 36, no. 3, pp. 517-536, 2002.

[13] N. B. Salah, A. Soulaimani, and W. G. Habashi, "A finite element method for magnetohydrodynamics," Computer Methods in Applied Mechanics and Engineering, vol. 190, no. 43-44, pp. 5867-5892, 2001.

[14] J.-F. Gerbeau, "A stabilized finite element method for the incompressible magnetohydrodynamic equations," Numerische Mathematik, vol. 87, no. 1, pp. 83-111, 2000.

[15] R. Codina and N. Hernández-Silva, "Stabilized finite element approximation of the stationary magneto-hydrodynamics equations," Computational Mechanics, vol. 38, no. 4-5, pp. 344-355, 2006.

[16] M. Gunzburger and C. Trenchea, "Optimal control of the time-periodic MHD equations," Nonlinear Analysis: Theory, Methods \& Applications, vol. 63, pp. 1687-1699, 2005. 
[17] S. H. Aydin, A. I. Nesliturk, and M. Tezer-Sezgin, "Two-level finite element method with a stabilizing subgrid for the incompressible MHD equations," International Journal for Numerical Methods in Fluids, vol. 62, no. 2, pp. 188-210, 2010.

[18] U. Hasler, A. Schneebeli, and D. Schötzau, "Mixed finite element approximation of incompressible MHD problems based on weighted regularization," Applied Numerical Mathematics, vol. 51, no. 1, pp. 19-45, 2004.

[19] D. Schötzau, "Mixed finite element methods for stationary incompressible magneto-hydrodynamics," Numerische Mathematik, vol. 96, no. 4, pp. 771-800, 2004.

[20] S. Gao and H. Duan, "Negative norm least-squares methods for the incompressible magnetohydrodynamic equations," Acta Mathematica Scientia B, vol. 28, no. 3, pp. 675-684, 2008.

[21] P. Houston, D. Schötzau, and X. Wei, "A mixed DG method for linearized incompressible magnetohydrodynamics," Journal of Scientific Computing, vol. 40, no. 1-3, pp. 281-314, 2009.

[22] C. Greif, D. Li, D. Schötzau, and X. Wei, "A mixed finite element method with exactly divergence-free velocities for incompressible magnetohydrodynamics," Computer Methods in Applied Mechanics and Engineering, vol. 199, no. 45-48, pp. 2840-2855, 2010.

[23] A. Prohl, "Convergent finite element discretizations of the nonstationary incompressible magnetohydrodynamics system," Mathematical Modelling and Numerical Analysis, vol. 42, no. 6, pp. 1065-1087, 2008.

[24] L. Baňas and A. Prohl, "Convergent finite element discretization of the multi-fluid nonstationary incompressible magnetohydrodyn.amics equations," Mathematics of Computation, vol. 79, no. 272, pp. 1957-1999, 2010.

[25] M. Crouzeix and P. A. Raviart, "Conforming and nonconforming finite element methods for solving the stationary Stokes equations. I," RAIRO: Numerical Analysis, vol. 7, no. R-3, pp. 33-76, 1973.

[26] R. Rannacher and S. Turek, "Simple nonconforming quadrilateral Stokes element," Numerical Methods for Partial Differential Equations, vol. 8, no. 2, pp. 97-111, 1992.

[27] Z. Cai, J. Douglas Jr., and X. Ye, "A stable nonconforming quadrilateral finite element method for the stationary Stokes and Navier-Stokes equations," Calcolo, vol. 36, no. 4, pp. 215-232, 1999.

[28] Q. P. Deng, X. J. Xu, and S. M. Shen, "Maximum norm error estimates of Crouzeix-Raviart nonconforming finite element approximation of Navier-Stokes problem," Journal of Computational Mathematics, vol. 18, no. 2, pp. 141-156, 2000.

[29] D. Y. Shi and Y. R. Zhang, "A nonconforming anisotropic finite element approximation with moving grids for Stokes problem," Journal of Computational Mathematics, vol. 24, no. 5, pp. 561-578, 2006.

[30] D. Shi and J. Ren, "Nonconforming mixed finite element approximation to the stationary NavierStokes equations on anisotropic meshes," Nonlinear Analysis: Theory, Methods $\mathcal{E}$ Applications, vol. 71, no. 9, pp. 3842-3852, 2009.

[31] S. Zhang, X. Xie, and Y. Chen, "Low order nonconforming rectangular finite element methods for Darcy-Stokes problems," Journal of Computational Mathematics, vol. 27, no. 2-3, pp. 400-424, 2009.

[32] D. Shi and J. Ren, "Nonconforming mixed finite element method for the stationary conductionconvection problem," International Journal of Numerical Analysis and Modeling, vol. 6, no. 2, pp. 293-310, 2009.

[33] D. Shi and J. Ren, "A least squares Galerkin-Petrov nonconforming mixed finite element method for the stationary conduction-convection problem," Nonlinear Analysis: Theory, Methods E Applications, vol. 72, no. 3-4, pp. 1653-1667, 2010.

[34] G. Matthies and F. Schieweck, "Nonconforming finite elements of higher order satisfying a new compatibility condition," Journal of Numerical Mathematics, vol. 16, no. 1, pp. 23-50, 2008.

[35] D. Shi and L. Pei, "Low order Crouzeix-Raviart type nonconforming finite element methods for the 3D time-dependent Maxwell's equations," Applied Mathematics and Computation, vol. 211, no. 1, pp. 1-9, 2009.

[36] D. Shi and L. Pei, "Low order Crouzeix-Raviart type nonconforming finite element methods for approximating Maxwell's equations," International Journal of Numerical Analysis and Modeling, vol. 5, no. 3, pp. 373-385, 2008.

[37] V. Girault and P. A. Raviart, Finite Element Methods for Navier-Stokes Equations Theory and Algorithms, vol. 5 of Springer Series in Computational Mathematics, Springer, New York, NY, USA, 1986.

[38] R. Temam, Navier-Stokes Equations, vol. 2, North-Holland Publishing, Amsterdam, The Netherlands, 1984.

[39] T. Apel, S. Nicaise, and J. Schöberl, "Crouzeix-Raviart type finite elements on anisotropic meshes," Numerische Mathematik, vol. 89, no. 2, pp. 193-223, 2001. 
[40] C. Park and D. Sheen, " $P^{1}$-nonconforming quadrilateral finite element methods for second-order elliptic problems," SIAM Journal on Numerical Analysis, vol. 41, no. 2, pp. 624-640, 2003.

[41] Z. X. Chen, Finite Element Methods and Their Applications, Springer, Berlin, Germany, 2005.

[42] Q. Lin, L. Tobiska, and A. Zhou, "Superconvergence and extrapolation of non-conforming low order finite elements applied to the Poisson equation," IMA Journal of Numerical Analysis, vol. 25, no. 1, pp. 160-181, 2005.

[43] D. Shi, J. Ren, and X. Hao, "A new second order nonconforming mixed finite element scheme for the stationary Stokes and Navier-Stokes equations," Applied Mathematics and Computation, vol. 207, no. 2, pp. 462-477, 2009.

[44] D. Shi and C. Wang, "A new low-order non-conforming mixed finite-element scheme for secondorder elliptic problems," International Journal of Computer Mathematics, vol. 88, no. 10, pp. 2167-2177, 2011.

[45] D. Shi, J. Ren, and W. Gong, "A new nonconforming mixed finite element scheme for the stationary Navier-Stokes equations," Acta Mathematica Scientia B, vol. 31, no. 2, pp. 367-382, 2011.

[46] P. G. Ciarlet, The Finite Element Method for Elliptic Problems, vol. 4, North-Holland Publishing, Amsterdam, The Netherlands, 1978. 


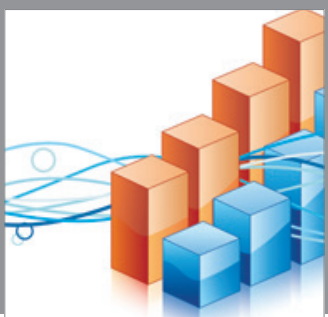

Advances in

Operations Research

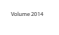

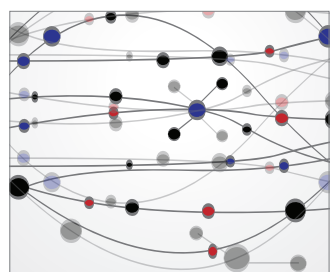

\section{The Scientific} World Journal
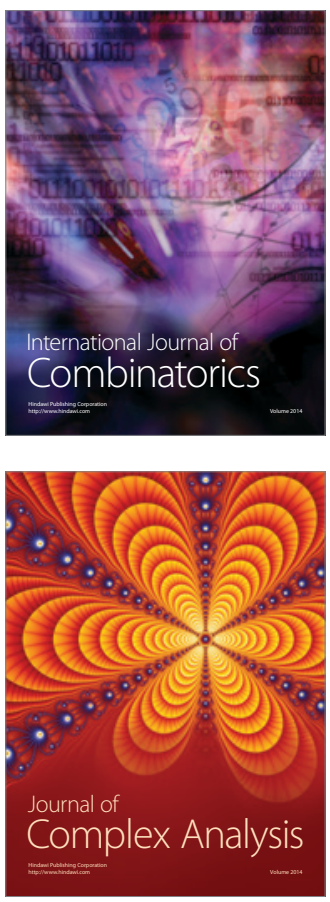

International Journal of

Mathematics and

Mathematical

Sciences
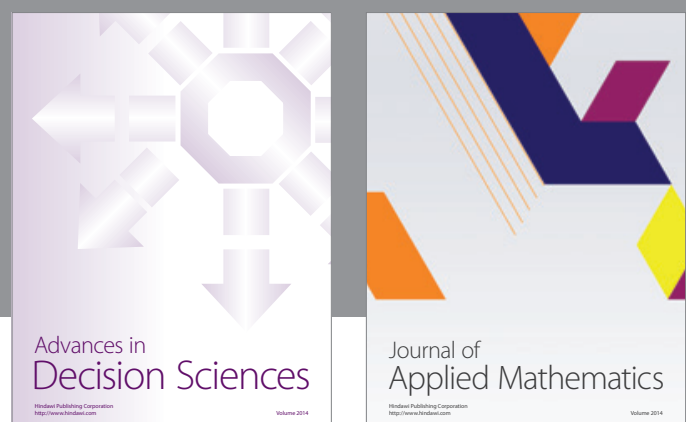

Journal of

Applied Mathematics
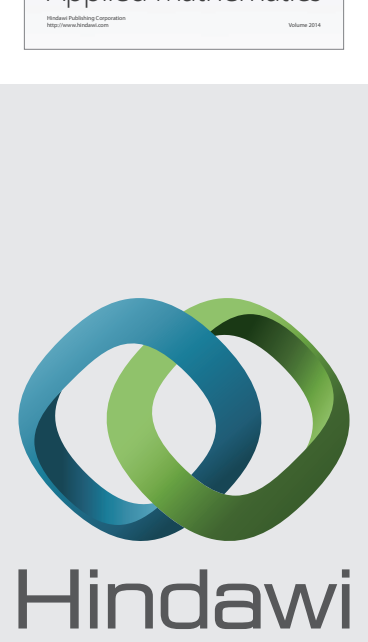

Submit your manuscripts at http://www.hindawi.com
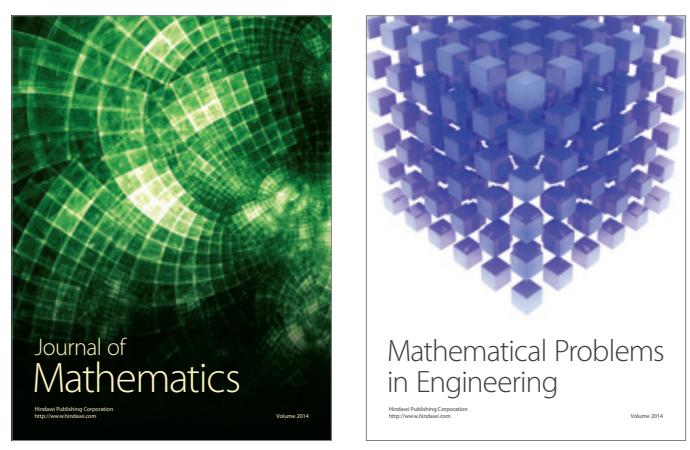

Mathematical Problems in Engineering
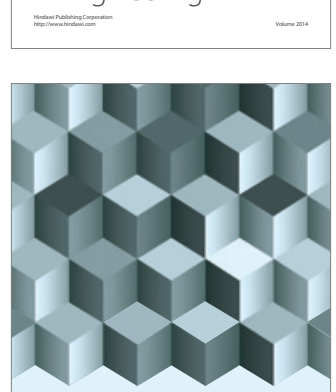

Journal of

Function Spaces
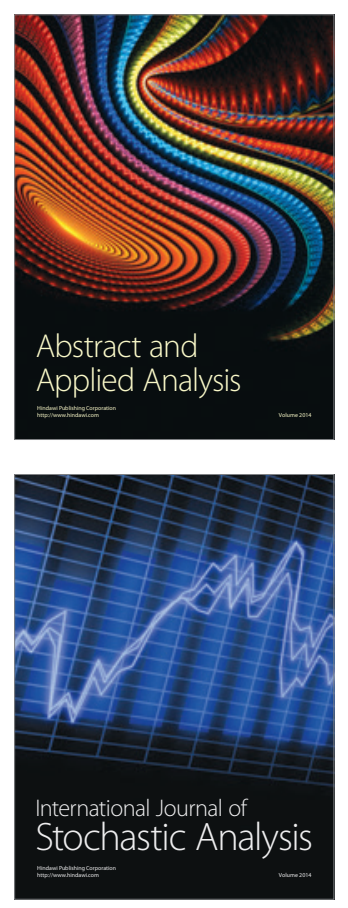

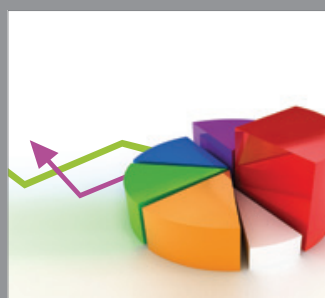

ournal of

Probability and Statistics

Promensencen
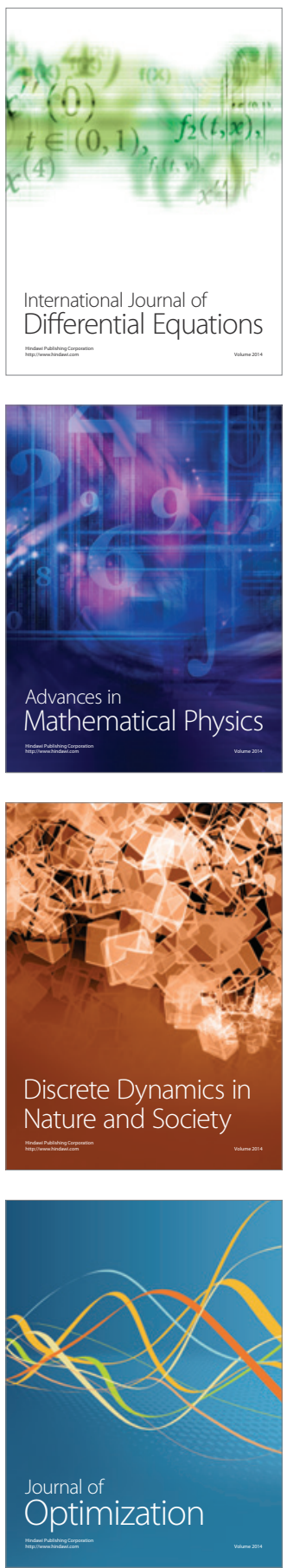